\title{
The Magic Jacket: Recognition and Organizational Psychology
}

\author{
Luke Strongman ${ }^{1}$ \\ ${ }^{1}$ Open Polytechnic, Lower Hutt, New Zealand \\ Correspondence: Luke Strongman, Communications, Open Polytechnic, 3 Cleary Street, Waterloo, Lower Hutt, \\ 5040, New Zealand. E-mail: Luke.Strongman@OpenPolytechnic.ac.nz \\ Received: November 9, 2016 \\ Accepted: November 30, 2016 \\ Online Published: December 14, 2016 \\ doi:10.5539/ijps.v9n1p33 \\ URL: http://dx.doi.org/10.5539/ijps.v9n1p33
}

\begin{abstract}
Recognition is essential in human social life. It is also critical in the workplace as one of the central communication activities that provides social cohesion, meaning and direction amongst colleagues and clients. Without expressions of recognition to others-formal and informal, high-context and low-context, social and structural, from a simple greeting to an affirmation for competent achievement, the workplace and the human behavior in it may become less than optimal and even dysfunctional. Expressions of recognition promote social cohesion. Based on a literature review and qualitative analysis, this article will provide an understanding of recognition in the workplace from a variety of viewpoints. It will explain recognition as central to the rationale of productivity, identify characteristics of its use and prevalence, discuss recognition as forms of behavioral guidance and social capital and exchange, before concluding to emphasize the role of recognition in the social and regulative functions of the modern workplace.
\end{abstract}

Keywords: recognition, job evaluation, organisational culture, human relations, employee engagement, continuous professional development, organisational psychology

\section{Introduction and Definitions of Recognition}

Recognition in human relations goes beyond advertisement. Businesses advertise to promote their services and to gain clients. Some do so on the basis of the attraction of the goods they offer; others on the skill of the services they provide. To ensure they keep their custom, most business aim to treat their clients politely and with respect. However, sometimes business neglect their staff communication at the expense of their communication with clients. Recognition is not always about the marketed product. Most companies are focused on providing effective communication for their business dealings with clients; but fewer organizations have a similar emphasis on their communications with internal staff. Those that do usually have an internal communication culture that is more professionally coordinated and which leads to enhanced motivation and staff performance. The way in which an organization "talks" to its staff and in which staff are encouraged to talk to one-another can have a large impact on the attitude and ultimately on the performance of the organization and the employees within it (Warman, 2004, p. 70). Recognition of self and others is not of itself an egocentric activity, although it is important to well-being, as it forms a social regulative function.

Affirmation and social support are essential for the internal communication in an organization and both stem from the same fundamental communication source-recognition. Employees and employers alike almost always desire to be accepted and everyone likes to be told that they are doing well or doing a good job (Olson, 2008, p. 16). But the unfortunate truth is that many organizational cultures (through lack or professional training or engagement) default to "deficit" models of communication, in which employees are more often told what they are doing wrong, rather than what they are doing right. There are three varieties of misrecognition that occur. Firstly, resistance, either an individual or colleagues do not identify them with recognition, or the available recognition schemes are unaligned with aspirations and personal appraisal of learning; secondly marginalization whereby and individual's aspirations are not recognizable within setting's participatory opportunities; and, thirdly, rejection whereby, participatory opportunities are incompatible with an individual's capabilities, leading to experience of or active mutual rejection of possible recognition (p. 357). An everyday organizational climate of "guilty until proven innocent" can also take its toll on workplace morale. Positive recognition as reward for work done, is however, conducive of a productive and engaging cultural climate. 
Because of the hectic pace of day-to-day business, or because people are more often than not dealing with problem areas, organizations and managers forget to recognize employees. But as Olson (2008) suggests recognition when it is sincere and appreciative goes a long way to reward employees and to promote organizational cohesion. Positive recognition can take many forms, from a one end of the spectrum, simply acknowledging another person through a facial expression, to being socially consistent, voicing a friendly greeting, giving praise, personal thanks. An employer giving job recognition such as a commendation or raise, are two relatively commonplace expressions of recognition. Management of "recognition" is about identifying those who need help but also about praising those who are excelling. For any employee or colleague, paying attention to another person, elaborating on what he or she did well, taking an interest in families without meddling and respecting privacy are all traits of positive recognition in the workplace that go a long way to enhancing organizational culture and producing optimal performance amongst staff.

\section{Methodology}

This article engages in qualitative analysis on the topic of workplace recognition. A literature survey was undertaken on relevant topics in organisational and workplace recognition in the prevailing scientific discourse with a subject focus on recognition in human relations, and organisational psychology literature. Findings from the scientific literature were then analysed and organised into focal areas which are then discussed taking into account salient concepts from the practice or neglect of organisational recognition and its effect on workplace morale and productivity. The strength of the study is that the approach is a broad qualitative survey analysis based on current literature which allows the consolidation of salient areas of enquiry into generalizable inferences about workplace practices in recognition. The limitation is that while assessments on workplace behaviours and organisational impacts are useful, and specific suggestions made for improving workplace practice, each workplace context in which organisational recognition takes place is necessary idiosyncratic. Nevertheless, broad and generic inferences can be made about the relevance of workplace recognition to organisational morale and productivity.

\section{Productivity Rationale}

Without recognition workplace productivity may decline through an erosion of positive morale. Hence, a productivity rationale underpins recognition in the workplace, though it does not provide the only reason for recognition practices in the workplace. Molla (2009) suggests that practicing recognition is an expected part of management behavior and if organizations don't practice recognize recognition then they don't advance (p. 14). Organizations work well when achievements are celebrated but most of all when the value of recognition is central to the organization, when it is tied to business objectives, integrated into the organizational plan, is discussed in management meetings, included in written communications, and recognition celebration held frequently. Thus recognition in these activities is a more integral part of an organizational culture and extends a philosophy of the social practice of a "pat on the back" to more tangible measures of workplace achievement. Sandberg and Kubiak (2013) argue that: "Recognition is necessary for self-realisation ... [the] ... use of this term refers to the fundamental formation of self and identity as a person with value and autonomy" (p. 352). Not only is recognition valuable for workplace morale, it is also a form of social affirmation and goodwill that affects well-being.

In most organizations recognition is an organizational practice, something that is done, not something constituted by the organization itself. However, most organizations can strive to "do recognition" better (Watson, 2014). Organizations can create cultures of recognition, and most organizations' culture in its simplest form is a way of doing things that is derived from organizational values, and is observed from the practices and behaviors of its members, even though these are values may be inferred rather than directly seen or explicitly stated (Watson, 2014). There are a wide range of possible recognition behaviors in an organization, a vital part of which are "intangible" best understood as ideas. These ideas are negotiated between learning opportunities, choices, and increased responsibilities. So recognition is an ascription of value to an activity but most of all a person responsible for the activity. It has optimum efficacy when made consistently to acknowledge or reward high performance, rather than inconsistently to meet short-term targets.

As Nelson (2003) argues many companies talk about recognition but a "culture of recognition" is hard to establish. In any organization employees are the most important resource (despite reliance on technology and stock). Some believe that recognition is a human right and for an organization to work well, everyone must acknowledge that recognition has a positive impact on employee morale, tenure, performance and productivity (p. 13). Unfortunately, many organizations do not "do" recognition well because it is seen as not an intrinsically self-centered activity, and difficult for others carry-out given priorities in working arrangements, although it is 
highly advantageous for organizational purposes. Companies and organizations that do recognize the achievements of their staff well have recognition as an integral part of their culture. As such, in large organizations, recognition may be mentioned in value statements, be a part of strategic personnel plans, and be an expectation of management engagement with staff. However, organizations have to work continually to keep recognition "alive" in the minds of employees (Nelson, 2003, p. 13). When a culture of recognition is dysfunctional recognition is perceived of as inequity or favoritism.

\section{Recognition at Work Schemes}

Many organizations adopt "recognition schemes" that reward and acknowledge employees for their achievements. One way of improving recognition schemes in the workplace is to ask questions about the way in which recognition is measured and managed? What are the business conditions in which recognition schemes are most often used? Is the practice bottom-up or top-down driven? Are recognition schemes values driven and centrally orientated? Are recognition schemes formal or informal, how is meaning shared amongst them? How does an employer know that there is a correlation between recognition, motivation, increased performance and compensation?

In the contemporary workplace there is a trend towards variable compensation scheme systems, flexible working arrangements, that involve telecommuting and video-conferencing for meetings, for example, all of which require some form of recognition behaviors. Most managers agree that recognition of employees enhances performance, that recognition schemes involve staff in providing practical feedback, that it may enable good work to get done, and that some forms of recognition may also enhance the personal goals of employees within the organization (and even give them confidence outside it). Most studies concur that recognition is most effective when it is made soon after the causing event or assessment rather than later from it. The incidence of recognition schemes in the workplace is reasonably high but it may not be ingrained in many management practices. As Li, Harris, Zheng, and Liu (2016) state, recent estimate-three out of four every four organizations use formal recognition programs (p. 925). Such systems primarily involve non-financial, symbolic gestures of appreciation, which have incentive types tailored to individual preference (p. 925). Li et al. note that it is possible that “ ... formally identifying and recognizing a top performer may boost other teammates' productivity via key social influence processes ...” (p. 925). However, competition can sometimes provide negative stimulus for those who are unsuccessful in gaining the reward of recognition. Social influence has a powerful effect on recognition levels within an organization. Both role modelling recognition and social learning of recognition behavioral repertoires and responses can produce a positive spill-over effect in which a performer who is recognized formally may enhance a recipient teammate's individual and collective performance (Li et al., 2016, pp. 925-926). Such recognition events are also acknowledgement of organizational values and messages of affirmation and reinforcement (Li et al., 2016, p. 926). Hence it behoves organizations to identify "carrots" amongst the motivational sticks, with which to incentivize employee engagement.

A defining attribute of self-other relations is relatedness which brings people together in behaviors that involve recognition of one-another. As such it seen that recognition occurs both in an "intimate sphere" of individuals and "public sphere" of groups and politics. Although the sense it is employed in most organizations belongs to an individuated sense of a collective identity, as a system of reward it is often focused primarily at individual level, although how national, ethnic groups are viewed or valued becomes a cumulative factor of culturally embedded recognition behaviors (Castleman, 2016, p. 139).

Recognition of oneself (through reflective practices) and recognition of others through reflective practices is related to behaviors of respect though it is conceptually distinct because it is based in inherent human values, that are grounded in notions of dignity. All human interactions affect ideas and perceptions of dignity. Positive self-other relations in which positive recognition of others is received is understood to increase a person's sense of dignity. By comparison a negative recognition decreases human dignity.

Given that most people within organizations are embedded in complex social systems which have reciprocal influences, incentive in the form of recognition may improve positive normalizing and socializing functions. In this context it is relevant to note that social influence is seen as a process of information sharing and learning in which employees selectively determine informational relevance and can assign behavioral utility to others. Recognition schemes may provide strong tangible signals to others in valuing behaviors and conferring legitimating roles on recipients (Li et al., 2016, p. 927). Furthermore, recognition is on a spectrum of formality or informality. As Li et al. (2016) note, people constantly make active and passive observation of others, and at times people make vicarious assessments of others as a part of ordinary behavior. Social ties permit or prohibits the observation of others, network patterns, the proximity of neighbors and in group and out-group behaviors. 
The centrality of people within the network of workflow amplifies the positive effect of recognition on team performance which also influences those on the periphery (p. 927).

\section{Peer Recognition Schemes}

Of the various recognition methods, peer recognition has a powerful effect on staff morale and motivation. Recognition is meaningful when it comes from colleagues and schemes that are based on the idea that staff can nominate their colleagues for a reward and management can decide from the nominations which is more deserving are not costly in terms of resources and promote good communication and motivation. Peer recognition schemes promote organizational values and responsibility. If peer recognition schemes are linked to business objectives and avoid being popularity contests they are more effective (Coleman, 2010, p. 31). Some peer recognition schemes are based on revenue and targets, they are cross-functional and collaborative, and some give employees a choice of reward. Although receiving an award from peer nomination can boost morale, not everyone thrives on being in the limelight (Coleman, 2010, p. 32). Other forms of peer recognition than social popularity and approval need to be considered, if peer recognition schemes are formalized in the workplace.

Organizations rely on staff commitment, and one of the largest expenses in an organizational culture is recruitment and staff change, hence promoting a cohesive culture through forms of recognition is an important part of organizational well-being. As Coleman (2010) suggests, recognizing staff performance by reward increases motivation but recognition from peers is more often than not valued more highly, both result in organizational gains from staff loyalty and retention (p. 31).

\section{Recognition and Behavioral Guidance}

Recognition can be both a verbal and a non-verbal communication behavior or expression. As a form of behavioral practice it can have remarkable effects on morale and motivation within organizations. As well as positive psychological effects there are those that derive from basic affinity as human beings. As Castleman (2016) states, "Human recognition is defined as the extent to which an individual is acknowledged by others to be of inherent value by virtue of being a fellow human being" (p. 135). A fundamental constant throughout all ethnicities of the is that membership of the human race is what people have in common. It is a part of their shared identity. Furthermore, aspects of human self-determination theory have a eudemonic conception of well-being that recognizes the integrity of perceiving others as self-like and acknowledging and rewarding the efforts and successes of others. For many people it is comforting to know that there are others like them, and that sometimes being acknowledged is a way of encouraging social participation and promoting cohesion. Recognition can be either positive by actively acknowledging the value of an individual as being a human being who has something in common with other human beings; or negative, referring to viewing an individual as lacking inherent value as a human being or ignoring or denigrating his or her value. By contrast, negative human recognition can involve dehumanization and objectification (Castleman, 2016, p. 135). Treating others with respect is a form of values-sharing.

\section{Dehumanization and the "Still-Face" Paradigm}

Dehumanization refers to the deprivation of human or individual qualities, while objectification refers to treating a person as a commodity or product devoid of dignity or personality. In situations where recognition is lacking there is a loss of contingent interaction as the unexpected combination of other's gaze and unresponsiveness play an important role. The cessation of vocal cues, facial cues, and tactile simulation in social interaction leads to despondency. In comparison, eye contact signals openness to interaction but problems arise in the display contradictory information or in situations where there are mixed signals, for example, if a care-giver is communicating "hello" and "goodbye" simultaneously. Care-givers and parents are important modulators, and if supportive social interaction is not available than some elements of socializing affinity may be lost. Along with care-givers colleagues and managers are also contributors to forms of recognition and social affinity in the workplace.

An important research finding in the absence of consistent positive recognition modulation by care-givers for infants is the Still-Face-Paradigm (SFP). This was first introduced by Tronick, Als., Adamson, Wise and Brazelton (1978) to test the hypothesis that infants are active contributors to social interaction. In their conceptualization of the "Still-Face Paradigm", infants are generally observed in a three-step face-to-face interaction with an adult: Firstly, a baseline normal interaction episode; secondly, the "still-face" episode in which the adult becomes unresponsive and maintains a neutral facial expression; and thirdly, a reunion in which the adult resumes normal interaction. Responses to neutral or no-communication produce a decrease in fidelity and morale, and increase likelihood of accurate emotional well-being. Positive emotional recognition stimulates 
human sharing, warmth, empathy and well-being from an early age. So too in the workplace, from an expression of greeting to a positive facial recognition behavior.

\section{Recognition and "State of Consciousness"}

People need positive external regulators to achieve optimal arousal levels, but show disorganization of emotion and behavior if a regulator is not available. These may effect an individual's state of consciousness. "State of Consciousness" (SOC) is described as an individual's continuously developing knowledge of the world and his or her relationship to the world. This state is psychobiological as it encompasses a complex organization of body, brain, behavior and experience. In a state of consciousness there are usually a spectrum of positive and negative thoughts and behaviors. Recognition of others can be distinguished between the general and the specific. That is, recognizing the inherent dignity and shared value of human beings generally, and secondly, valuing an individual because of qualities and skills which he or she possesses. Human recognition may refer both to the valuing of an individual and his or her treatment in a social or workplace context (although the second factor is contentious because an individual can be treated well but be simultaneously devalued for utilitarian reasons, or an individual can be treated badly but still be highly valued as a human being).

\section{Doing Recognition-Recognition of "Self" and "Other"}

Acknowledgement and sympathy are basic motivations for other-regarding qualities in human behavior beyond an instrumental purpose. Arguably in the modern world it is a workplace "service" to be observed, to be attended to and to be taken notice of with sympathy, if not at some level a human need or a "right" (Castleman, 2016, p. 136). Although recognition of others may set aside instrumental values and belong more to those of ontological harmony, the regard of and from fellow human beings is a fundamental motivation and objective of normal economic behaviors (Offer, 1997). Sharing values and rewarding effort is a basic part of mutuality and information exchange, a fundamental quantum of communication. A persistent factor of non-market exchange is peoples' pursuit of regard involving a range of positive interactions which might include: acknowledgement, attention, and respect. As such, "other regarding behaviour" fosters and maintains relationships and reputation - this is held to be a "good in its own right". Recognition of others has direct psychological benefits that are beyond instrumentalism of goal-setting behaviors (Castleman, 2016, p. 136).

\section{Recognition as a Form of Social Capital}

Aldrich (2012) notes that "social capital" namely the "networks, norms, and social trust that facilitate coordination and cooperation for mutual benefit". As Ross and Carter (2011) explain, "[t]he spontaneous activation of 'social capital' - bonds within and between social groups - [is] impressive, a solace to those affected, as well as a huge practical and economic benefit" (p. 2). "Human capital" is the ability of people to remain co-operative and resilient in communities and to work in a co-ordinated way together on issues in common, and also to celebrate common interests and to acknowledge and reward good behaviour. As such, social capital is an enabler of trust (Castleman, 2016, p. 142) and like human recognition is relational, involving interactions among individuals and groups. Dimensions of recognition are both material and psychological and can directly and indirectly influence well-being (Castleman, 2016, p. 143).

In terms of psychological effects, the level and quality of human recognition that is received by a person directly affects their well-being, independently of material outcomes (Castleman, 2016, p. 143). A person who is acknowledged and recognized by others is more likely to feel "in-tune" with the environment, and more likely to be both connected and harmonized. A person who is ignored is more likely to retreat socially, to be less self-expressive and to feel out of tune with the environment. He or she is less likely to seek reciprocal interaction and may be more prone to self-regulative thoughts. Such psychological effects of recognition or its absence can have real "material effects" on behaviors and actions for example which in turn affect other outcomes such as workplace morale, health, status, income and education.

Given that there is a subtle but tangible effect that the level of recognition can have on the actions and behaviors of both the individual providing recognition and the material well-being of the person who receives the recognition, then there is a behavioral causal relationship between other-regarding "healthy" and "less healthy" behaviors (Castleman, 2016, p. 144). Furthermore, these impacts are sometimes expressed through behavioral access towards opportunities and services. Social recognition may have both individual factors and a shared value horizon and common standard of judgement but nevertheless make individual differences socially visible (Fassauer \& Hartz, 2016, p. 178). Organizations that are attractive or prestigious are more likely to foster seducement to enhance a person's self-esteem but this self-esteem may be derived from an external source (Fassauer \& Hartz, 2016, p. 178). As any form of identity forming process, recognition may produce both 
enhanced effect (when positive) and degraded affect (when negative) and often variations between (Fassauer \& Hartz, 2016, p. 178).

Sometimes expectations of recognition are met and sometimes they are not. Arguably, highly inviting monetary rewards may be insufficient for producing positive relation to self over time because most involve some sacrifice to some notions of self in process (Fassauer \& Hartz, 2016, p. 190). Establishing "ethical moments" emerging relation-to-self address position in world such as in conferral or withholding of recognition is on a continuum of emotional experiences (Fassauer \& Hartz, 2016, p. 190). A way of mitigating this issue is for employees to ensure congenial working conditions and also for improving material development outcomes involving higher levels of recognition. There is a "fork" of complementarity that results. Individuals who receive higher levels of positive recognition are more likely to provide positive recognition to others; those that receive lower levels of recognition are likely to provide similar levels to others (Castleman, 2016, p. 145). Honneth (1996) regards recognition as: firstly, positive affirmation of a person's characteristics; secondly, an attitude realized in concrete action; and thirdly, distinct phenomenon of social world - result of side - effect of an action. However, there are differences between forms of recognition and levels of both affect and perception. For example, "love" may be a form of mutual care and affection and also "formalized" in a relationship of "cognitive-rational" affect between legally equal partners. It may be distinguished from legal recognition, in which subjects are autonomous and morally responsible, and distinguished from rights-based recognitions derived from legal status and charters.

\section{The Co-Agency of Workplace Recognition}

Employers and employees are in a relationship of agency and responsibility, to each-other, the organization, and its clients and stakeholders. As Fassauer and Hartz (2016) argue there are "various instances of agency" which aim to affect institutional order (p. 173). As Sandberg and Kubiak (2013) relate, in many workplaces recognition is expressed through "tangible affordances of engagement" in participatory practices, work tasks, opportunities for decision making. Recognition is "generally understood as a positive evaluative response to a person's behaviors, actions, and identities" (Fassauer \& Hartz, 2016, p. 173). Recognition occurs along a dimension of identity-forming social interaction. Recognition impacts behaviors by affecting emotions which result in both changes and maintenance of organizational arrangements (Fassauer \& Hartz, 2016, p. 173). For Ekman (2013) there is an ideological pursuit of social recognition in which relationships between managers and employees satisfy the "hunger for validation" in mutual subjectification and perpetuation of power structures. For Creed et al. (2014) there are "sharing attempts" which are pursued by institutionalized communities. For Fassauer and Hartz (2016) institutional offers of recognition have "seductive" character and are tied to relations of mutual recognition, identity and respect. Identity recognition can give feeling of "security". But organizational recognition, which scrutinizes employers' and employees' desires for identity, can oppress aspirations towards self-realization (p. 173). However, there is a "potential" or recognition that can be a stimulant for change. Recognition and motivation can also be engaged in forms of institutional work that are apart from rational interest. Satisfaction of rational interests might be significant but not sufficient to engender enduring feelings of self-respect (Fassauer \& Hartz, 2016, p. 175). Struggles for recognition, can produce both social advancement and critical views of organizations. As such, "proactive germane self-realization" calls for mutual recognition, as a general driver of ongoing social development and organizational achievement (Fassauer \& Hartz, 2016, p. 176). So try to practice the simple question with your colleagues, "How are you today?"- -a simple question but vital for the workplace - you never know where it might lead.

\section{Conclusion}

Based on qualitative analysis from a literature review, this article has provided a broad overview of considerations, factors and impacts from an organisational psychology perspective on workplace recognition. The article has provided definitions of "recognition" in the workplace, examined the role of recognition in the productivity rationale underpinning company organisation; discussed recognition in the workplace as peer recognition schemes, discussed recognition as a moderator of behaviour and its impact on "state of consciousness". The role of "recognition" as a motivator and in the enhancement of social capital, is also discussed as well the co-agency of recognition behaviours in the workplace. While the present study is limited in the specificity of its focus to specific workplace contexts, it is nevertheless grounded in the rigorous discussion of from current scientific literature of issues affecting a broad range of organisations and companies. The emergent general inference is that workplace recognition has a direct bearing on organisational morale and productivity, aside from its expression as a core part of civil behaviour. 


\section{Funding information/Conflict of Interest Declarations}

There are no funding sources that have a direct or indirect political interest on the article. There are no conflict of interest disclaimers.

\section{Acknowledgements}

I am grateful to Dr Raymond Young and the staff of the communication section at the Open Polytechnic for their consultation.

\section{References}

Aldrich, D. P. (2012). Social, not physical, infrastructure: The critical role of civil society after the 1923 Tokyo earthquake. Disasters, 36(3), 399-400. https://dx.doi.org/10.1111/j.1467-7717.2011.01263.x

Castleman, T. (2016). The role of human recognition in development. Oxford Development Studies, 44(2), 135-151. https://dx.doi.org/10.1080/13600818.2015.1109615

Coleman, A. (2010). Local Heroes. Employee Benefits, 31-32.

Creed, W. E. D., Hudson, B. A., Okhuysen, G. A., \& Smith-Crowe, K. (2014). Swimming in a sea of shame: Incorporating emotion into explanation of institutional reproduction and change. Academy of Management Review, 39(3), 275-301. https://dx.doi.org/10.5465/amr.2012.0074

Ekman, P. (Ed.). (1998). Charles Darwin's “The Expression of the Emotions in Man and Animals", Third Edition, with Introduction, Afterwords and Commentaries. New York: Harper-Collins/Oxford University Press.

Fassauer, G., \& Hartz, R. (2016). Stories of Adoration and Agony: The Entanglement of Struggles for recognition, Emotions and Institutional Work. Schmalenbach Business Review, 17, 173-193. https://dx.doi.org/10.1007/s41464-016-0015-6

Honneth, A. (1996). The Struggle for Recognition: The Moral of Social Conflicts (Joel Anderson, Trans.). Massachusetts: The MIT Press.

Li, N., Harris, T. B., Zheng, X., \& Liu, X. (2016). Recognizing "Me" Benefits "We": Investigating the positive Spillover Effects of Formal Individual Recognition in Teams. Journal of Applied Psychology, 101(7), 925-939. https://dx.doi.org/10.1037/ap10000101

Mesman, J., van Ijzendoorn, M. H., \& Bakermans-Kranenburg, M. J. (2009). The many faces of the Still-Face Paradigm: A review. Developmental Review, 29(2), 120-162. https://dx.doi.org/10.1016/j.dr.2009.02.001

Molla, T. (2009). The three R's. Fleet Equipment, 35(12), 5.

Nelson, B. (2003, February 1). Put their names in lights. In Meetings Net. Retrieved from $\mathrm{http}: / /$ meetingsnet.com/corporate-meetings/put-their-names-lights

Offer, A. (1997). Between the gift and the market: The economy of regard. The Economic History Review, 50(3), 450-476. https://dx.doi.org/10.1111/1468-0289.00064

Olson, J. (2008). Recognition. Surface Fabrication, 14(1), 16.

Tronick, E., Als, H., Adamson, L., Wise, S., \& Brazelton, T. B. (1978). Infants response to entrapment between contradictory messages in face-to-face interaction. Journal of American Academy of Child and Adolescent Psychiatry, 17, 1-13. https://dx.doi.org/10.1016/S0002-7138(09)62273-1

Warman, D. (2004). Out box opinion. Employee Benefits, 70.

Watson, B. (2004, April 14). Developing a Culture of Recognition. ABA Bank Marketing, 14.

Zhao, W., Chellappa, R., Phillips, P. J., \& Rosenfield, A. (2003). Face Recognition: A Literature Survey. ACM Computing Surveys, 35(4), 399-458. https://dx.doi.org/10.1145/954339.954342

\section{Copyrights}

Copyright for this article is retained by the author(s), with first publication rights granted to the journal.

This is an open-access article distributed under the terms and conditions of the Creative Commons Attribution license (http://creativecommons.org/licenses/by/4.0/). 